\title{
Le temps des boucliers
}

\section{François Lissarrague}

\section{(2) OpenEdition}

\section{Journals}

Édition électronique

URL : http://journals.openedition.org/imagesrevues/850

DOI : 10.4000/imagesrevues.850

ISSN : 1778-3801

\section{Éditeur :}

Centre d'Histoire et Théorie des Arts, Groupe d'Anthropologie Historique de l'Occident Médiéval, Laboratoire d'Anthropologie Sociale, UMR 8210 Anthropologie et Histoire des Mondes Antiques

\section{Référence électronique}

François Lissarraque, « Le temps des boucliers », Images Re-vues [En ligne], Hors-série 1 | 2008, mis en ligne le 21 avril 2011, consulté le 30 janvier 2021. URL : http://journals.openedition.org/imagesrevues/ 850 ; DOI : https://doi.org/10.4000/imagesrevues.850

Ce document a été généré automatiquement le 30 janvier 2021.

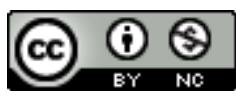

Images Re-vues est mise à disposition selon les termes de la Licence Creative Commons Attribution Pas d'Utilisation Commerciale 4.0 International. 


\title{
Le temps des boucliers
}

\author{
François Lissarrague
}

1 Dans le système des images grecques, le bouclier constitue un 'objet théorique' remarquable sous le triple aspect de la temporalité, de la transmission et de la tradition de l'image.

Il doit cette importance en premier lieu à son statut d'objet technique : le bouclier hoplon - fait le guerrier, l'hoplite ${ }^{1}$. Il forme un maillon de la chaîne défensive qui unit

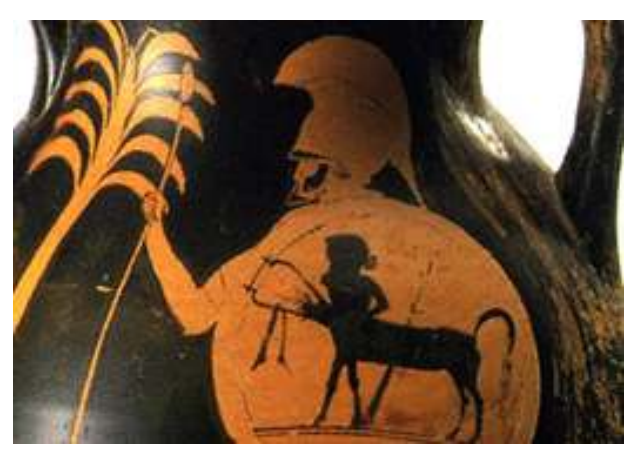
entre eux les hoplites en rang serré face à l'adversaire; chaque bouclier est comme une écaille d'une carapace qui constitue l'armée en corps homogène. Par ailleurs, pris isolément, chaque bouclier fait partie du corps du guerrier, et l'abandon du bouclier signifie le renoncement à soi-même. On connaît la formule que prononce une mère spartiate remettant un bouclier à son fils ${ }^{2}$ : «Reviens avec lui ou sur lui », c'est-à-dire en vainqueur, avec le bouclier, ou mort, gisant sur ce même bouclier, devenu civière. En aucun cas le guerrier ne doit se séparer de cette arme identitaire, sous peine de déchoir.

Mais le bouclier n'est pas seulement l'arme qui permet de faire face à l'ennemi, de tenir son rang et de présenter à l'armée adverse un mur de métal. Le bouclier est porteur d'image ; il est à lui seul une image et fait l'objet d'un fort investissement iconique dans l'histoire culturelle grecque.

De ce point de vue, le bouclier d'Achille est primordial. Dans l'Iliade, on s'en souvient, Achille humilié par Agamemnon refuse de prendre plus longtemps part aux combats et se retire sous sa tente. Devant les difficultés des Grecs face aux assauts ennemis, il accepte de prêter ses armes à Patrocle qui s'en revêt et repousse alors les Troyens, terrifiés à la vue de celui qu'ils prennent pour Achille de retour. Les armes font en partie l'identité de celui qui les porte. Mais Hector affronte cet Achille-Patrocle, le tue et le dépouille; il se revêt à son tour de ces armes. Corps et identité se confondent en partie, pas totalement, et les armes circulent, par appropriations successives. L'armure, 
la panoplie, fonctionne comme un second corps mais elle connait une temporalité et des formes de circulation distinctes de celles qui rythment le corps héroïque ${ }^{3}$.

5 A ce point de l'Iliade, Achille est désarmé, mais décidé à venger Patrocle. Il lui faut une nouvelle armure qui ne peut être que divine. C'est l'objet du chant 18 ; Thétis se rend chez Héphaïstos qui forge à sa demande une nouvelle panoplie. L'épisode fait l'objet d'une ample focalisation descriptive, centrée non pas sur la panoplie complète, mais avant tout sur le bouclier ${ }^{4}$. La description que donne Homère du décor complexe est devenue dans l'histoire littéraire le modèle de l'ekphrasis, de la description d'une œuvre d'art. En fait le poète ne décrit pas l'objet, il raconte le processus de sa fabrication, la mise en forme de cet ensemble complexe d'images, où se déploient la cité en paix et la cité en guerre ${ }^{5}$. La complexité et l'abondance de ces scènes rendent impossible leur réalisation concrète, et il n'existe, quoiqu'on en ait dit, aucun équivalent matériel antique de ce bouclier de mots ${ }^{6}$. Seuls quelques modernes, admirateurs ambitieux de la beauté antique, tels Flaxman, se sont risqués à réaliser cette ekphrasis, sans avoir le talent ni d'Héphaïstos, ni d'Homère ${ }^{7}$.

Fig.1

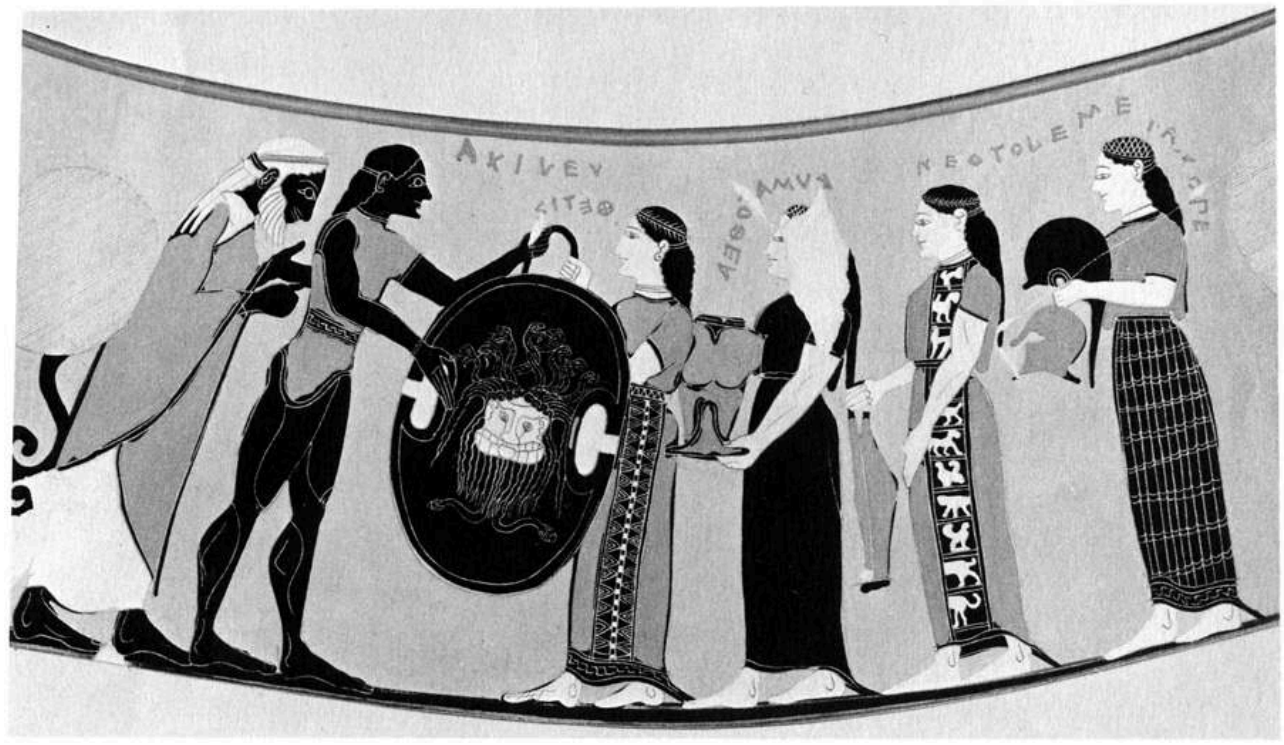

Boston, amphore fn, Remise des armes Thétis Achille

dessin d'après $\mathrm{CB}$

$6 \quad$ Les peintres de vases n'ont pas eu cette audace, et aucun des boucliers d'Achille connus par la céramique n'atteint une telle complexité. Leur stratégie, visuelle et non verbale, est différente. Pour évoquer ce même épisode de la seconde armure d'Achille, ils choisissent de montrer le moment où Thétis, accompagnée des Néréides, apporte sa nouvelle panoplie au jeune héros. L'image déploie alors l'ensemble des armes, comme sur une amphore tyrrhénienne de Boston (fig. 1) $)^{8}$ où les éléments sont portés horizontalement, en commençant par le bouclier, suivi de la cuirasse, des cnémides puis du casque : au total un corps en morceaux. On notera que le bouclier est présenté par Thétis non pas de profil vers Achille, mais de face, de manière à exhiber face au spectateur le visage de la Gorgone qui couvre tout le bouclier.

7 Le Gorgoneion fonctionne en effet, comme l'a bien montré F. Frontisi-Ducroux, à la manière d'un prosopon', une face toujours frontale, face de terreur et d'épouvante, dont 
l'original mythique pétrifie qui la regarde. La coïncidence entre le Gorgoneion et le bouclier n'est pas seulement liée à un choix d'efficacité apotropaïque ; elle est fondée dans le mythe par la liaison entre le bouclier-miroir et la décapitation de Gorgo. Dans ce récit, le bouclier sert d'interface pour neutraliser le pouvoir de Gorgo. Un tel dispositif optique met le reflet au centre du bouclier, qui devient ainsi le lieu de la Gorgone, le Gorgoneion au sens strict, (comme on dit le Theseion ou l'Olympeion), une sorte de temenos de Gorgo, un lieu de la représentation, un espace découpé, circulaire où s'inscrit cette figure de mort.

Fig.2

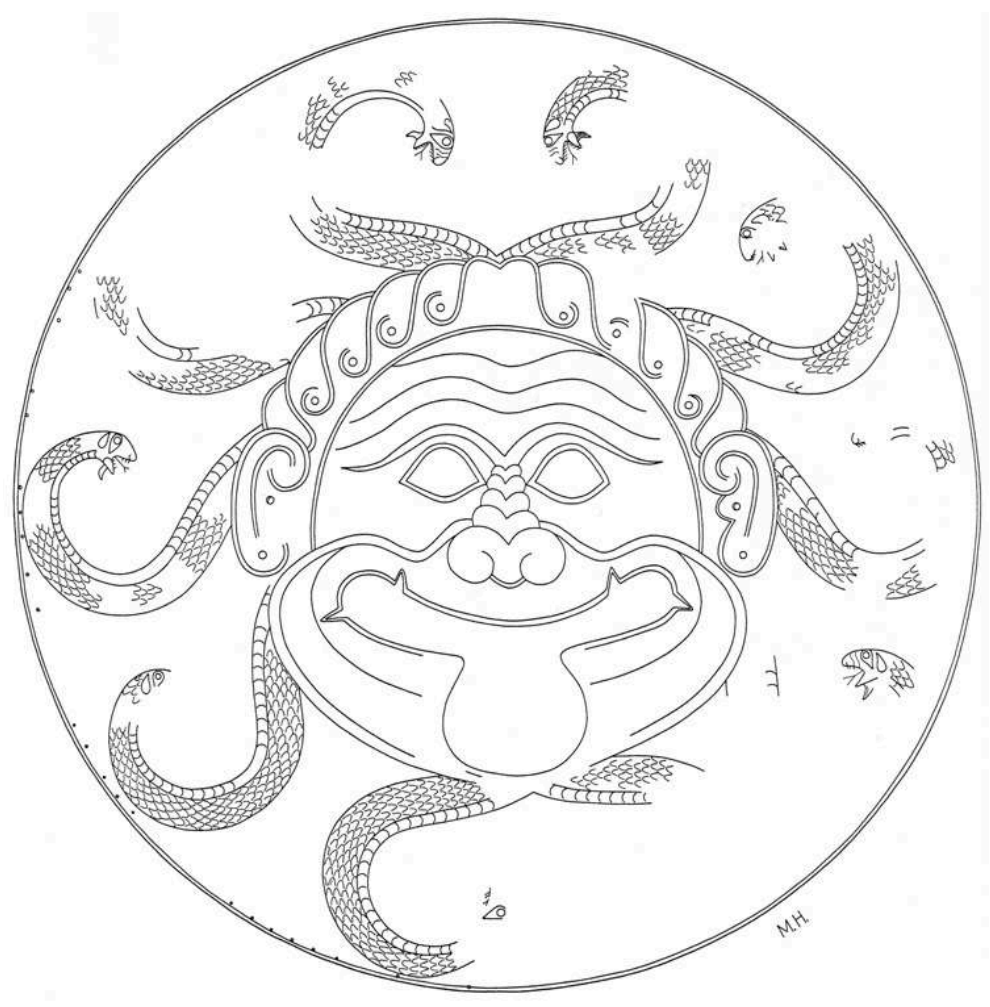

Olympie, bouclier votif , Gorgoneion

dessin d'après OF 
Fig.3

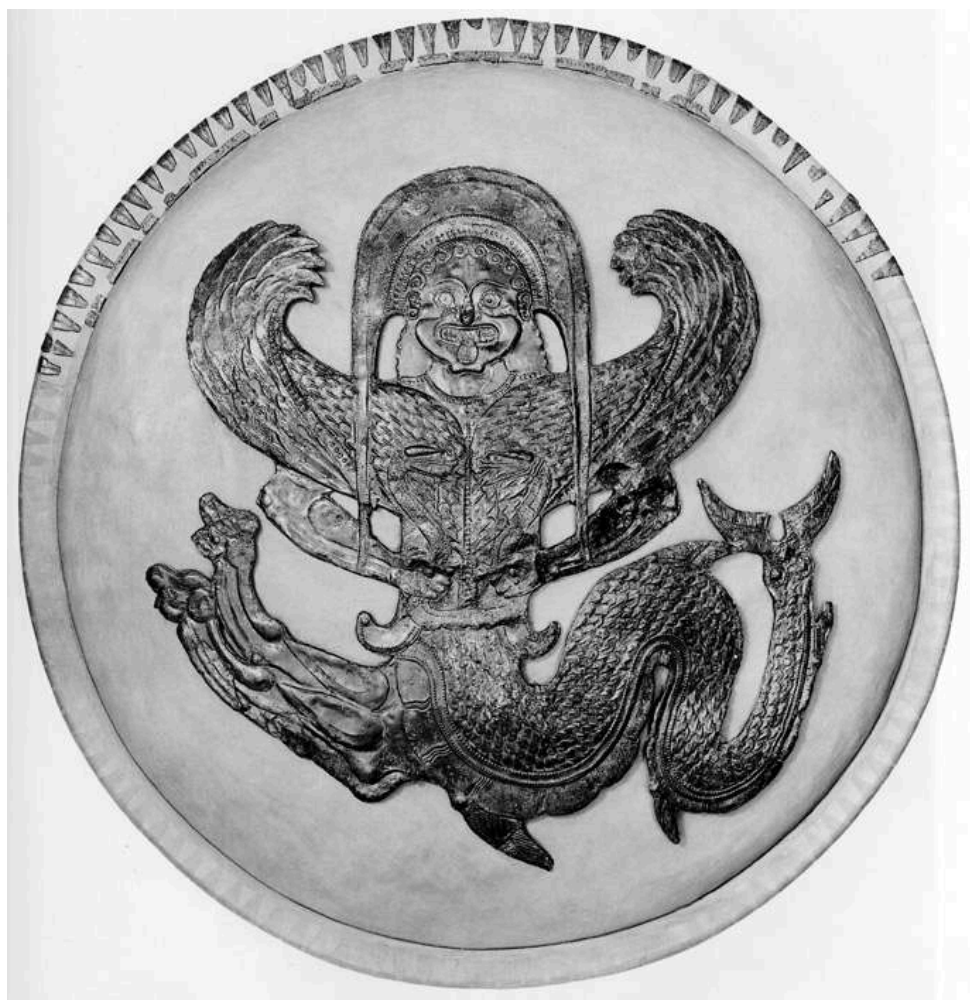

Olympie, bouclier votif, Gorgone casquée

dessin d'après OF

On voit à quel point le bouclier est pensé comme image, aussi bien dans la tradition de l'ekphrasis que dans l'iconographie archaïque, où les exemples de bouclier à Gorgoneion abondent. Deux boucliers trouvés à Olympie confirment cet intérêt visuel pour le Gorgoneion. Le premier (fig. 2) ${ }^{10}$ inscrit la face de Gorgo au cœur du dispositif, et organise en rayons circulaires la chevelure de serpents qui accentue l'horreur monstrueuse de ce mixte, humain et animal. Un autre exemplaire (fig. 3) ${ }^{11}$ élabore une variante unique mais révélatrice. Gorgo y figure, non comme simple face, mais comme un corps hybride, monstrueux, où se mêlent, conformément à la logique mythique, un torse et des bras humains avec des pattes de félin, une queue de poisson et des ailes largement déployées. L'animalité ainsi construite couvre aussi bien la terre que l'air et l'eau. Quant à la face, frontale comme il se doit, elle est casquée, auréolée d'un large cimier en arc de cercle. La figure monstrueuse qui, en épisème, protège le guerrier devient ainsi figure d'un guerrier porteur d'épouvante; hoplite et gorgone sont en partie assimilés, celle-ci se faisant guerrière, et celui-là assumant l'épouvante qu'elle provoque. Le choix d'un tel emblème produit comme un effet d'osmose entre la figure du guerrier et l'image sur le bouclier. 
Fig.4

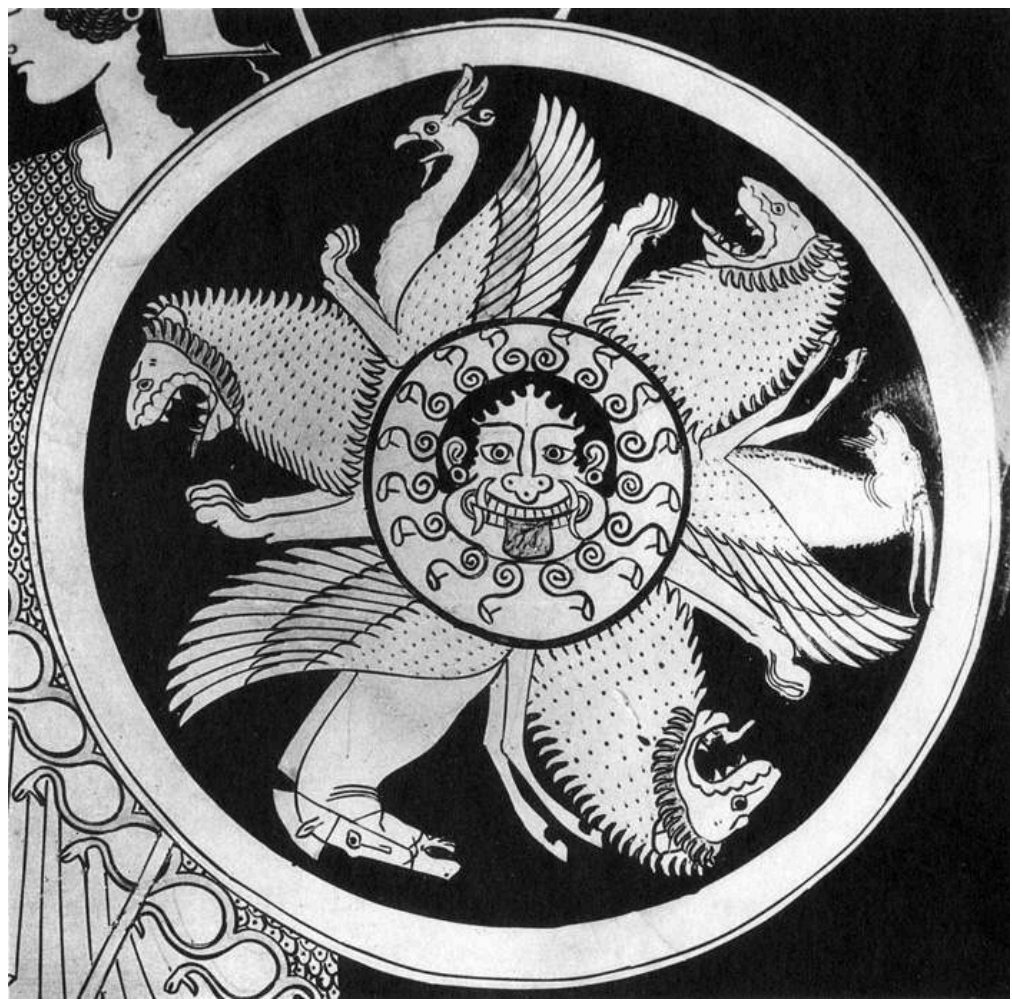

Bale, amphore fr., Détail du bouclier d'Athéna photo d'après AK

9 La céramique attique abonde en exemples de variations graphiques complexes autour de la face de Gorgo. Le jeu de l'hybridation par assemblage et combinatoire d'éléments hétéroclites a souvent séduit les peintres, comme le montre l'exemple du bouclier d'Athéna sur une amphore attribuée au peintre de Berlin (fig. 4) ${ }^{12}$. Le large bouclier de la déesse couvre son égide et dissimule le Gorgoneion qui s'y trouve habituellement; mais il amplifie ce même motif en le projetant sur le disque du bouclier. Le cœur du dispositif est occupé par la face frontale, bordée de serpents, puis entourée d'un cercle interminable qui tourne visuellement dans le sens inverse des aiguilles d'une montre, cercle formé de trois protomés de lions, alternant avec des monstres ailés : un griffon, un pégase, une chimère, aigle, cheval et chèvre ailés qui à nouveau associent l'air et la terre et donnent son dynamisme à ce mouvement circulaire.

Au-delà de ces jeux de variations autour du motif ancien du bouclier-gorgoneion, d'autres aspects des épisèmes méritent qu'on s'y arrête, en particulier les jeux temporels qu'ils permettent. Le système grec des blasons est distinct de ce que nous connaissons par l'héraldique médiévale. Celle-ci donne au bouclier une valeur identitaire, comme en Grèce, mais aussi une valeur généalogique. Le même blason se transmet de père en fils, manifestant ainsi une temporalité de la filiation; il se complexifie à travers les alliances, subdivisant et accumulant les motifs à travers un système d'engendrement des blasons qui reflète l'histoire des familles, comme l'a bien montré $\mathrm{M}$. Pastoureau ${ }^{13}$. Dans le cas grec, les épisèmes sont beaucoup plus libres et ne font pas l'objet d'une transmission codifiée comme dans l'Occident médiéval. La variété des motifs est grande, si l'on examine par exemple le répertoire des boucliers consacrés à Olympie ${ }^{14}$. Elle est encore plus grande en image, car les peintres jouent librement des 
possibilités graphiques que permet la représentation, sur chaque bouclier, d'une image à l'intérieur de l'image. La présence de ces cercles inclus au sein d'un ensemble figuratif plus large permet des jeux d'interférence entre plusieurs plans: celui de l'image englobante et celui de l'épisème englobé, ou bien d'un bouclier à l'autre, par des jeux d'écho, de répétition ou de complémentarité.

11 L'image mise en cercle, sur le bouclier, permet aussi des variations chromatiques, des changements d'échelle, des segmentations qui viennent interférer avec l'image englobante. Beaucoup de peintres se contentent de répéter des motifs courants, sans aller plus loin, mais certaines images semblent bien porter la marque d'un travail très conscient sur les possibilités qu'offre ce type d'assemblage et d'inclusion, enrichissant ainsi la dialectique visuelle entre le bouclier et son porteur, l'image emblématique et l'identité du guerrier. Il existe quelques exemples de boucliers dont l'emblème dit le nom de son porteur ; ainsi sur une coupe attribuée au peintre de Nicosthénès ${ }^{15}$, le géant Kyknos, fils d'Arès porte un cygne (kyknos) sur son bouclier. Mais, pour Kyknos, cet exemple est unique, et l'on ne connaît pas beaucoup d'épisèmes parlants de ce genre ${ }^{16}$. Le rapport entre l'épisème et son porteur comporte parfois une dimension temporelle remarquable, associant à l'identité du guerrier soit une image qui renvoie à son passé, à un de ses exploits, soit au contraire une figure qui anticipe sur son avenir. L'épisème prend alors une valeur énonciative de type narratif ou prédictif. On ne peut se contenter, pour saisir la signification de ces blasons, d'en dresser une liste lexicale ${ }^{17}$, par types, en affectant à chaque type une valeur sémantique particulière. On doit tenir compte des configurations spécifiques que produit chaque image, et des interférences qui résultent de ces jeux d'inclusion.

La tragédie d'Eschyle, les Sept contre Thèbes, utilise de manière remarquable la valeur énonciative des épisèmes, comme l'a montré entre autres $\mathrm{F}$. Zeitlin ${ }^{18}$. Le messager, on s'en souvient, décrit à Etéocle, qui règne à Thèbes, les boucliers que portent chacun des Sept chefs conjurés en marche contre sa ville, et menés par Polynice, son frère ennemi. Chaque bouclier est un énoncé prédictif, une déclaration d'intention sur ce que fera celui qui le porte. L'un des boucliers porte même une inscription au futur "j'incendierai la ville ${ }^{19}$. Etéocle réagit à chaque description par un exercice d'interprétation et une décision qui répond à l'annonce ainsi déchiffrée. L'épisème est en quelque sorte prophétique et, en tant qu'oracle, doit être interprété. Dans le cas d'Hippomédon, qui a pour épisème Typhée, un des êtres primordiaux qui vise, après les Titans et les Géants, à prendre la place du roi des Olympiens, Etéocle répond en lui opposant Hyperbios dont le bouclier porte un Zeus. Il recrée ainsi au niveau des épisèmes la lutte Zeus-Typhée, qui apparaît alors comme le modèle mythique de la lutte entre Hyperbios et Hippomédon et préfigure l'échec souhaité du chef argien. Bien évidemment aucun des boucliers évoqués par Eschyle n'a d'existence archéologique ${ }^{20}$; ce sont des motifs qui ont leur rôle dans la logique interne de la tragédie, et font image, en paroles, à l'intérieur du spectacle théâtral. 
Fig.5

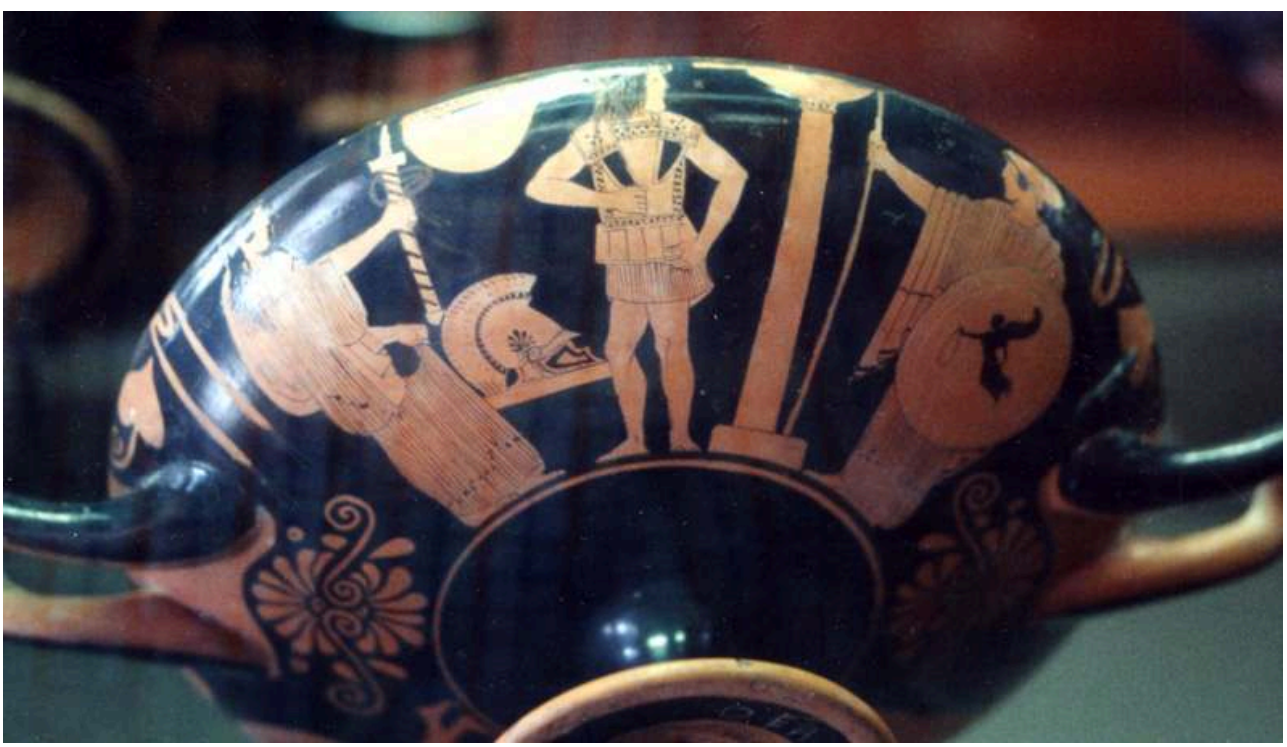

Louvre, coupe fr., Départ de guerrier

photo RMN

Une telle structure dramatique et visuelle se trouve déjà mise en œuvre par les imagiers ${ }^{21}$, sur certains vases qu i utilisent les épisèmes comme le moyen d'introduire dans l'image une épaisseur temporelle ou une dynamique spatiale. Sur une coupe du Louvre $^{22}$ (fig. 5), un guerrier prend les armes; il n'est pas encore entièrement équipé. Une femme à gauche lui tend une épée ; un casque est posé devant lui et une autre femme, à droite, lui apporte un bouclier où figure une Victoire en vol. La disposition du bouclier fait que la victoire vole vers le guerrier qui s'équipe; l'image en silhouette interfère avec la scène générale et la valeur prophétique d'un tel emblème ne fait guère de doute.

Dans d'autres cas, c'est avant tout le mouvement qui est privilégié. Plusieurs représentations d'hoplitodromes montrent un coureur dont le bouclier porte à son tour l'image d'un coureur. Simple effet d'écho, de mise en abyme qui intensifie la valeur de l'image et dont les peintres du début du $5^{\mathrm{e}}$ siècle sont friands. Mais parfois le coureur est immobile, encore dans ses préparatifs, alors que l'image sur l'épisème court déjà, pourrait-on dire ; le décalage est évident, et crée un effet dynamique incontestable ${ }^{23}$. 
Fig.6

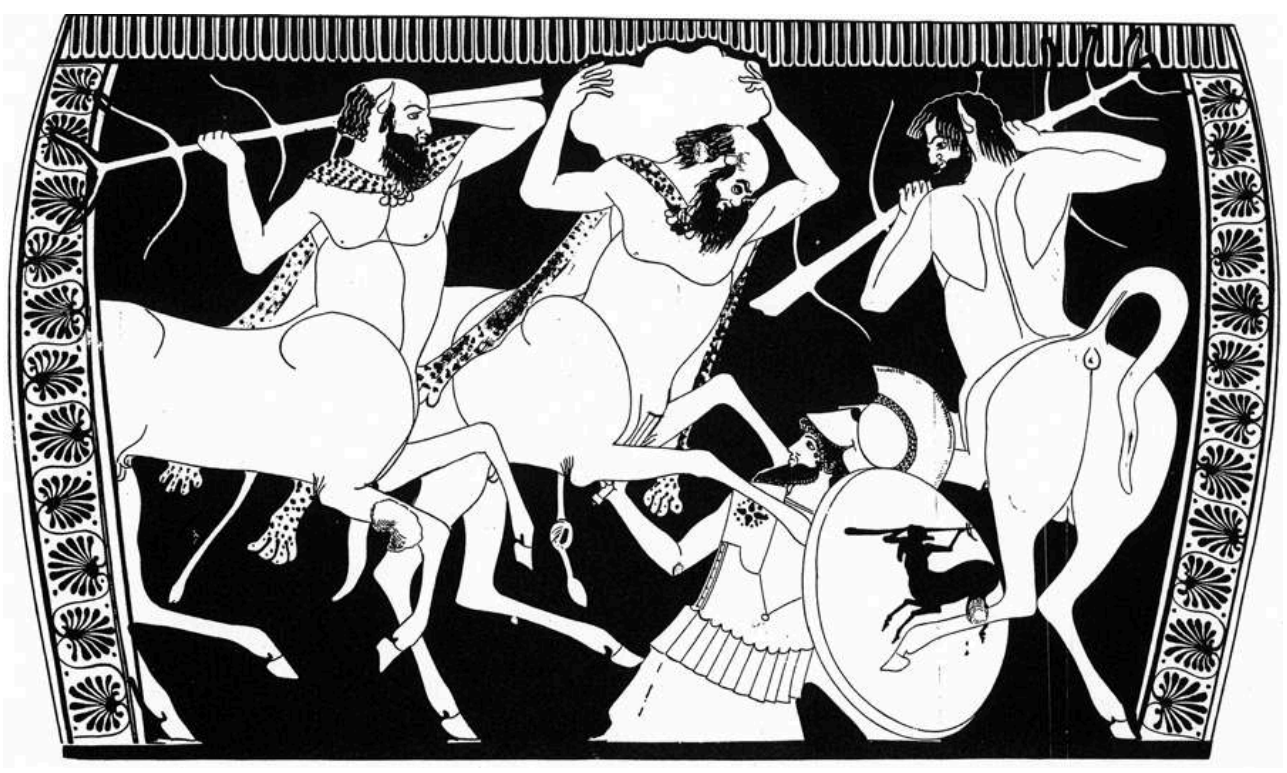

Harrow, cratère fr. ; Kaineus englouti par les Centaures

dessin d'après JHS 17, pl.16

Parfois les jeux d'interférence sont plus complexes. Sur un cratère de Harrow ${ }^{24}$ (fig. 6), trois Centaures achèvent d'enfoncer Kaineus dans le sol ; il est déjà enterré à mi-corps et lutte en brandissant un bouclier qui porte l'image d'un quatrième centaure. Ici encore se crée une sorte d'interférence entre les Centaures en action et l'image du centaure sur le bouclier de Kaineus, qui semble relayer la violence de ses congénères et boucler l'encerclement du héros.

Si l'on se place du point de vue purement visuel, le plan de l'image englobante et celui de l'image englobée se répondent, tout en maintenant un écart d'échelle et de chromatisme (le centaure du bouclier étant en silhouette noire, alors que les autres sont en figures rouges). Mais si l'on se place du point de vue de l'identité de Kaineus, on peut voir dans le centaure qu'il porte le signe de sa mort prochaine. Cette logique narrative, qui semble visuellement évidente, est paradoxale du point de vue de l'identité du porteur de bouclier. Comment souhaiter en effet porter le signe de sa propre mort et non celui de la mort de l'ennemi ? Avec ce cratère de Harrow, on voit bien comment, tout comme chez Eschyle, la logique ordinaire de l'épisème identitaire est travaillée et détournée au profit d'une logique dramatique, à destination non plus de l'ennemi face au bouclier, mais du spectateur face à l'image, du buveur face au cratère. 
Fig.7

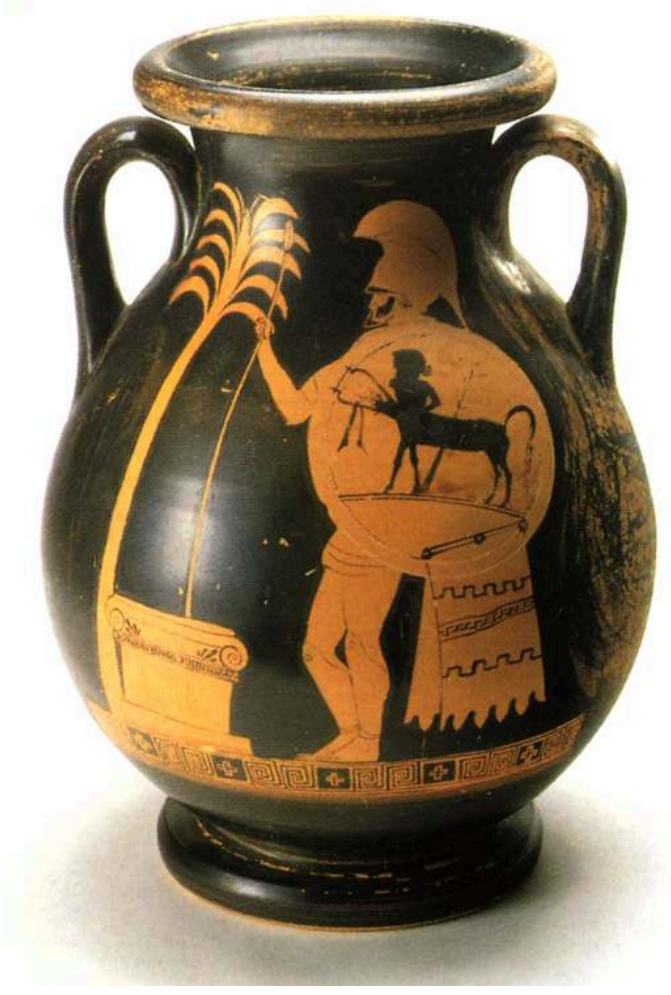

Commerce, péliké fr., Achille?

photo d'après LIMC

17 De même sur une péliké des années 470 (fig.7) ${ }^{25}$, un guerrier en arrêt devant un autel a pour épisème un centaure immobile, jouant de la lyre. Cette compétence musicale permet d'identifier le Centaure Chiron, seul musicien parmi ce monde de brutes ; et l'on peut alors penser que le guerrier pourrait être Achille, dont Chiron fut l'éducateur. Rien n'impose cette lecture, mais elle reste possible; si on l'accepte, on voit comment l'identité du héros est ici construite en référence à son histoire passée et au prestige de sa généalogie divine. 


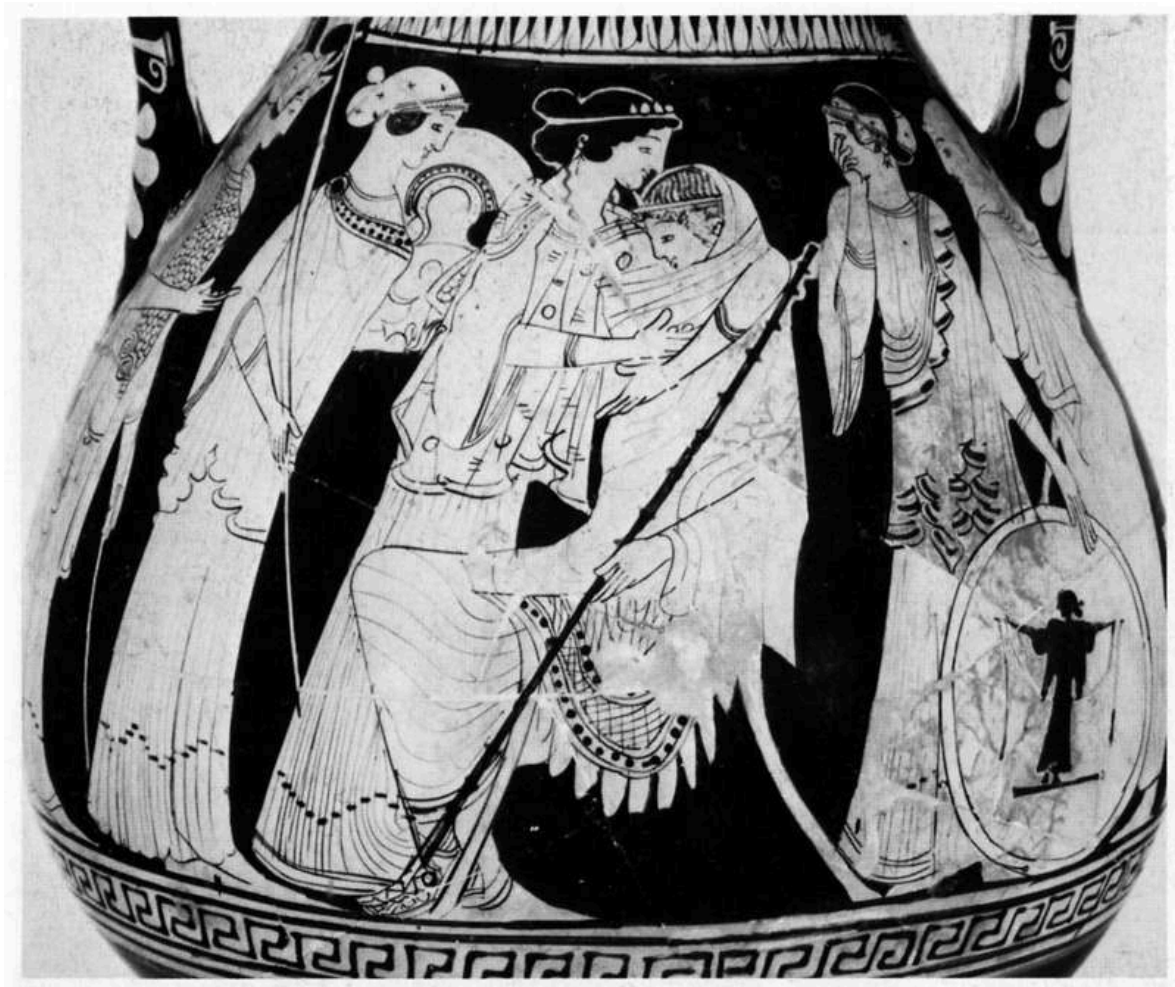

Londres, péliké fr., Bouclier d'Achille

photo d'après LIMC

Sur une autre péliké, à peu près contemporaine, c'est à coup sûr Achille qui figure au centre de la scène (fig. 8) ${ }^{26}$; assis, engoncé dans son himation qui le cache presque totalement, il est entouré de sa mère Thétis et de Néréides qui apportent ses armes. Sur le bouclier se détache la silhouette d'une femme filant. Paradoxe de ce motif qui renvoie à l'activité féminine par excellence, le filage, et nous entraîne loin des exploits héroïques. Mais l'image prend tout son sens si l'on se réfère à la biographie d'Achille, d'abord caché par sa mère chez le roi Lycomède, déguisé en fille et élevé comme tel parmi les filles du roi, afin d'échapper à son destin de guerrier. On sait comment cette ruse maternelle échoue : Ulysse déguisé en marchand apporte, cachées sous des tissus et des bijoux destinés aux jeunes filles du roi, des armes à la vue desquelles Achille se révèle pour ce qu'il est : le meilleur des Achéens. L'image incluse renvoie, semble-t-il, à cet épisode, au temps où Achille était encore une jeune fille, avant qu'il ne prenne pour de bon les armes et ne devienne le héros que l'on sait. 


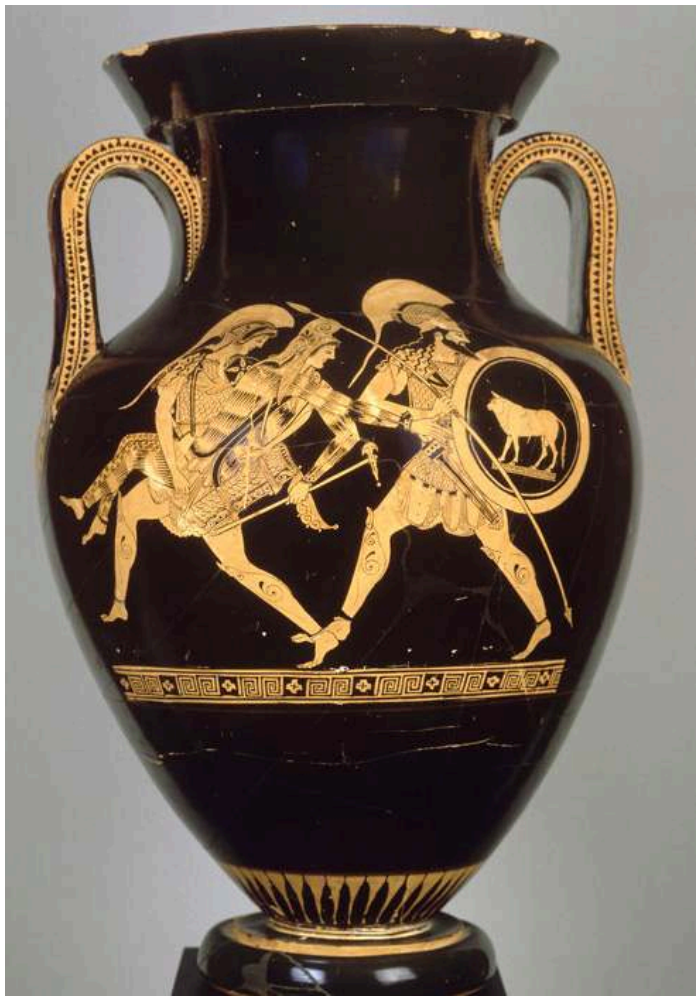

Louvre amphore fr., Thésée enlève Antiope photo RMN

D'autres boucliers renvoient ainsi au temps passé. Sur une amphore attribuée à Myson (fig. 9) ${ }^{27}$, Thésée enlève l'Amazone Antiope. Son compagnon Pirithoos porte un bouclier où s'inscrit, en figures rouges, un taureau. On pourrait, suivant une suggestion de Plutarque à propos des monnaies d'Athènes ${ }^{28}$, y voir une référence au taureau de Marathon, dont Thésée fut vainqueur. En ce cas, l'épisème inscrit dans l'image un exploit du passé qui fonde le prestige et la bravoure du jeune roi d'Athènes. L'image incluse apporte une référence qui enrichit le sens de l'image englobante. Elle n'est pas nécessaire, et le spectateur peut ne pas la reconnaitre ; mais si on la prend en compte sur ce mode, elle ajoute un écho temporel à l'image et contribue à son épaisseur sémantique.

De manière plus évidente, la référence au passé à travers l'image de l'épisème se déploie sur un petit groupe de trois amphores qui répètent le même choix iconographique. Il s'agit de trois amphores panathénaïques, c'est-à-dire de trois vases donnés en prix au vainqueur lors des concours en l'honneur d'Athéna. Ce sont des vases 'officiels' en quelque sorte. Ils portent une iconographie standardisée, contrôlée par les magistrats de la cité. D'un côté figure, entre deux colonnes, l'image de la déesse Athéna en position de combat, lance levée et bouclier porté en avant : une Athéna Promachos ; de l'autre côté est représentée l'épreuve correspondant à ce prix. Ce type d'amphore apparaît vers le milieu du VIe siècle, et se maintient, toujours dans le style à figures noires, jusqu'au IV siècle avancé2 ${ }^{2}$. Ces amphores portent fréquemment le nom de l'archonte qui règne cette année-là, et c'est pour nous un précieux élément de datation. Seules variantes, dans ce système fortement codifié : les motifs qui figurent en haut des colonnes d'encadrement et le blason de la déesse, qui varie pour chaque festival. 
Fig.10a

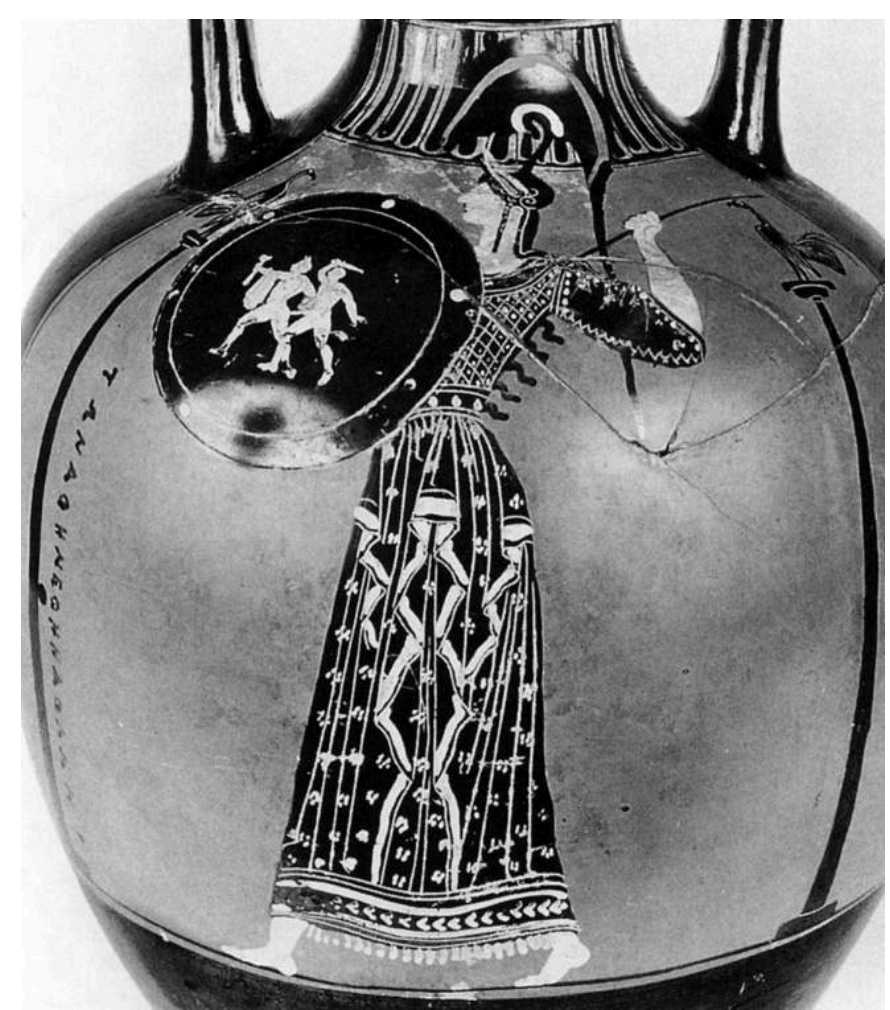

Hildesheim, amphore panathénaique fn ; Athéna promachos photo d'après Benz, pl. 97 
Fig.10b

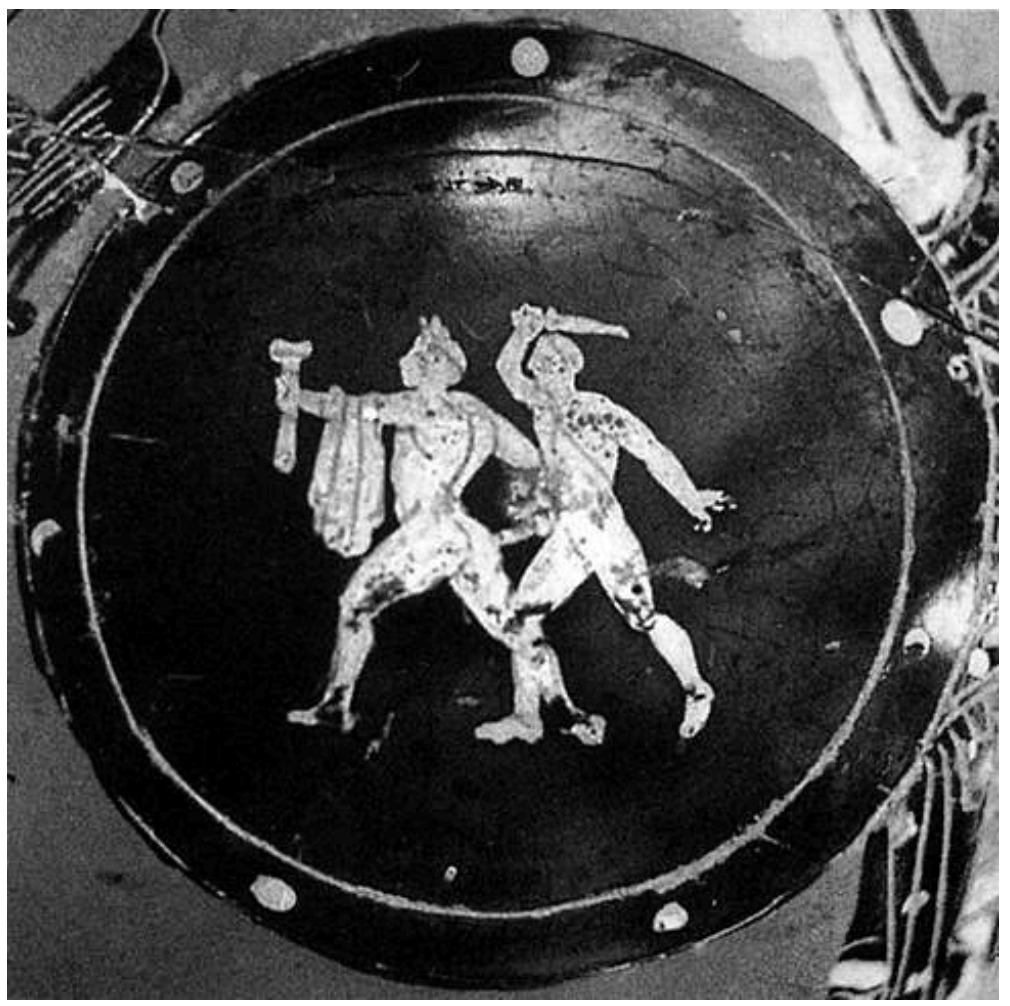

Hildesheim, amphore panathénaique fn ; Athéna promachos

photo d'après Benz, pl. 97

21 Sur trois de ces amphores, produites la même année, le bouclier de la déesse est orné d'un groupe de deux hommes, en silhouette blanche. Bras tendu, épée levée, ils sont dans une dynamique d'attaque qui prolonge le mouvement de l'Athéna Promachos. Ce groupe est connu par ailleurs: il s'agit des Tyranoctones, les deux Athéniens, Harmodios et Aristogiton, qui lors des Panathénées de 514 av. J.-C. assassinèrent Hippias, neveu d'Hipparque, tyran en exercice ${ }^{30}$. Aussitôt condamnés, ces deux citoyens reçurent des honneurs héroïques dès la chute de la tyrannie, en 510. Un groupe statuaire fut dressé sur l'agora d'Athènes, symbole de la liberté retrouvée. On en connaît diverses copies qui permettent de reconnaître ici, sur le bouclier d'Athéna, l'image des Tyranoctones, ou plus exactement l'image de la statue des Tyranoctones (fig. $10 \mathrm{a}$ et b). L'emblème fonctionne ici comme une citation ${ }^{31}$, à n'en pas douter, comme une image d'image, une image au second degré ${ }^{32}$. Ce jeu de référence produit une chaîne temporelle remarquable, dans l'identité de la cité et sa mémoire collective. Le groupe ici reproduit est en effet le second, celui qui a été élaboré par Critios et Nessiotès, pour remplacer un premier groupe dû à Anténor et emporté par Xerxès à Persépolis lors de l'invasion de l'Attique par les Perses en 480. Cet emblème, délibérément confisqué par l'ennemi pour ajouter à l'humiliation d'Athènes, fut aussitôt remplacé par un autre, le second groupe, visible sur l'agora pendant toute l'époque classique. C'est ce groupe qui figure sur le bouclier d'Athéna, lors des Panathénées de 400, après la chute des Trente tyrans. La réactivation de cette icône civique souligne la continuité voulue par les magistrats d'Athènes; en choisissant ce motif, ils construisent l'événement récent, l'expulsion des Trente, comme le miroir de l'évènement passé, la chute des Pisistratides. Ainsi l'histoire se répète, ou plutôt le 
discours civique met en évidence cette répétition, cette continuité. Athènes chasse toujours ses tyrans et le bouclier d'Athéna porte la marque de cette continuité, construite comme telle par les magistrats de la cité. En plaçant un tel emblème sur le bouclier d'Athéna, ils font du geste libérateur le signe de la force civique, l'identité de la déesse Promachos, combattante de la liberté passe par l'appropriation visuelle de l'histoire et de la mémoire collective des Athéniens. Le bouclier porte ici le signe de l'identité civique et politique au sens le plus large et se rapproche ainsi des stratégies iconographiques que l'on observe sur le monnayage athénien ${ }^{33}$.

Un tel cas est pour nous exceptionnel ; mais il est fortement significatif, car il pousse loin la logique de l'image et son insertion dans une temporalité à multiple niveaux. Les boucliers sur les vases nous réservent encore des surprises, si l'on veut bien y prendre garde.

\section{NOTES}

1. On associe fréquemment hoplon et hoplite; peut-être faut-il privilégier le pluriel, hopla, les armes en général, comme le suggèrent J. F. Lazenby et D. Whitehead, « The Myth of the Hoplite's hoplon », Classical Quarterly 46 (1996), p. 27-33.

2. Plutarque, Dits des Lacédémoniennes, Moralia 241f. Voir à ce sujet N. Loraux « La belle mort spartiate », Ktema 2 (1977) p. 111 (repris in Les expériences de Tirésias, Paris 1989, p. 84).

3. Sur cette question du rapport corps et armes, voir mon article à paraître, "Corps et armes ; figures grecques du corps héroïque » in P. Brulé et al. éd. Langages et métaphores du corps, Rennes.

4. Iliade, 18 ; la description du bouclier proprement dit occupe les vers $478-607$, alors que le reste de la panoplie est expédié en quelques vers 608-612.

5. Sur ce point voir en dernier lieu F. Frontisi-Ducroux, «Avec son diaphragme visionnaire. Iduesi prapidessi. Il. XVIII, 481. A propos du bouclier d'Achille », REG 115 (2002), p. 463-484.

6. Voir K. Fittschen, Der Schilde des Achilleus, Archaeologia Homerica, II N1, Göttingen, 1973.

7. Sur ces essais, voir Fittschen, o.c. p. et J. Boardman, The Archaeology of Nostalgia, Londres 2002, p. 167 et fig. 152.

8. Boston MFA 21.21 ; ABV 84/3, peintre de Camtar, vers 570 av. J.-C ; LIMC s.v. Achilleus $n^{\circ} 191$; pour l'ensemble de la série voir LIMC, s.v. Achilleus $n^{\circ}$ 186-205 et 506-541.

9. F. Frontisi-Ducroux, Du masque au visage, Paris 1995, p.65-75.

10. Olympie, H. Phillip, Archaische Silhouettenbleche und Schildzeichen in Olympia (Olympische Forschungen 30), Berlin 2004, $\mathrm{n}^{\circ}$ 31, p. 237-244, pl. 31-35.

11. Olympie, $O F \quad 30, n^{\circ} 22$, p. 198-216, pl.1 et 18-23.

12. Bâle Antikensammlung und Sammlung Ludwig, BS 456; ARV2 1634/1bis, peintre de Berlin. Voir J.D. Beazley, «An amphora by the Berlin Painter », AK 4 (1961) 49-67, en particulier pour l'épisème p. 58-67.

13. M. Pastoureau, Traité d'héraldique, $2^{\mathrm{e}}$ éd., Paris, 1993.

14. Voir $O F 30$ qui donne un catalogue de 105 boucliers.

15. Tarquinia RC 2066 ; peintre de Nicosthénès, ARV2 126/23.

16. De même trouve-t-on quelques 'types parlants' en numismatique : une rose à Rhodes, une ancre à Ancyre, etc. Voir F. Rebuffat, La monnaie dans l'Antiquité, Paris 1996, p.168. 
17. C'est ce que fait, de manière par ailleurs fort utile, G. Chase The Shield Devices of the Greek in Art and Litterature, reprint Chicago 1979 (original HSCP 1902).

18. F. Zeitlin, Under the sign of the shield, Rome 1982 ; voir aussi P. Viadal-Naquet, « Les boucliers des héros », in J.-P. Vernant et P. Vidal-Naquet, Mythe et Tragédie deux, Paris 1986, p. 115-147.

19. Eschyle, Les Sept contre Thèbes, vers 434 .

20. Cf. L. Lupas et Z. Petre, Commentaire aux Sept contre Thèbes, Bucarest 1981, p. 130.

21. Voir mon article "Looking at shield devices. Tragedy and Vase painting", in Visualizing Tragedy, Ch. Kraus, J. Elsner, S. Goldhill, H. Foley eds., Princeton 2007, p.151-164.

22. Louvre G 272 ; peintre de Sabouroff, ARV2 837/3.

23. Comparer, par exemple les deux médaillons de coupe Berlin F 2307, (peintre d'Antiphon, ARV2 341/77) et Leyde PC 89 (peintre d'Alkimachos, ARV2 533/62).

24. Harrow 50 ; peintre de Cleveland, ARV2 516/5.

25. Londres, Sotheby's 18/7/1985, n²12 ; LIMC s.v. Achilleus 832, pl. 138.

26. Londres, BM E 363, maniériste indéterminé, ARV2 586/36 ; LIMC s.v. Achilleus 515.

27. Paris, Louvre G197; Myson, ARV2 238/1.

28. Plutarque, Vie de Thésée, 25, 3, indique que le héros «frappa une monnaie où il fit graver un bœuf, soit à cause du taureau de Marathon,...soit pour inviter les citoyens à l'agriculture». Plutarque, comme souvent quand il pose une question de ce type, qu'elle soit grecque ou romaine, ne tranche pas.

29. Sur l'ensemble de cette série voir $M$. Benz, Panathenäische Preisamphoren. Ein athenische Vasengattung und ihre Funktion vom 6.-4. Jh. v. Chr., $18^{e}$ supplément AK, Bâle, 1998 ; pour les trois vases qui nous occupent ici, voir ses $n^{\circ} 5.239,5.244$ et 5.245, pl. 96-97 et p. 48-51 pour la question des épisèmes.

30. Voir S. Brunnsåker, The Tyrant-slayers of Kritios and Nesiotes : a critical study of the sources and restorations, Stockholm 1971 et B. Fehr, Les Tyrannoctones: peut-on élever un monument à la démocratie? Paris, 1989.

31. Voir F. Lissarrague, «Comment citer en image ? Quelques variations grecques » in C. DarboPeschanski éd., La Citation dans l'Antiquité, Grenoble 2004, p. 103-108 (où l'éditeur a malencontreusement oublié d'insérer les illustrations ; je saisis donc l'occasion de reproduire un des exemplaires de cette série : Hildesheim 1254, Benz 5.245). Sur la citation en image voir aussi C. Cousin, "Mention et citation dans l'imagerie antique ", in Hôs ephat', dixerit quispiam, comme disait l'autre... Mécanismes de la citation et de la mention dans les langues de l'Antiquité, Ch. Nicolas éd., Grenoble, 2006, p. 265-278, qui reprend le même exemple, tout à fait unique, il est vrai.

32. Voir W. Oenbrinck, Das Bild im Bilde. Zur Darstellung von Götterstatuen und Kultbilder auf griechischen Vasen, Francfort 1997 et M. De Cesare, Le statue in immagine. Studi sulle raffigurazioni di statue nella pittura vascolare greca, Rome 1997.

33. Voir L. Lacroix, Les représentations de statues sur les monnaies grecques, Liège 1949.

\section{INDEX}

Thèmes : céramique

Index chronologique : Antiquité

Mots-clés : art grecque, objet théorique, Iliade, armure, Gorgoneion 


\section{AUTEUR}

\section{FRANÇOIS LISSARRAGUE}

Né en 1947, ancien élève de rien du tout, actuellement directeur d'études à l'EHESS

(«Anthropologie et image : l'expérience grecque »), après avoir été professeur de lettres classiques, documentaliste au CNRS, puis chargé de recherches et directeur de recherches au même CNRS. Membre du Centre Louis-Gernet (CNRS/EHESS) depuis 1980. Auteur de divers ouvrages personnels (Un flot d'images : une esthétique du banquet grec, Paris 1987 ; L'Autre guerrier, Paris 1990 ; Vases grecs : les Athéniens et leurs images, Paris 1999) ou collectifs (Hommes, dieux, héros de la Grèce, Rouen 1982 ; La Cité des images, Paris-Lausanne 1984 ; Héros et dieux de l'antiquité (avec I. Aghion et C. Barbillon), Paris 1994 ; Les mystères du gynécée - avec P. Veyne et F. Frontisi-, Paris 1996) et d'une centaine d'articles portant principalement sur les problèmes grecs de l'image. 